\title{
A REMARK ON 'SOME NUMERICAL RESULTS IN COMPLEX DIFFERENTIAL GEOMETRY'
}

\author{
Kefeng Liu and Xiaonan Ma
}

\begin{abstract}
In this note we verify certain statement about the operator $Q_{K}$ constructed by Donaldson in [3] by using the full asymptotic expansion of Bergman kernel obtained in [2] and [4].
\end{abstract}

In order to find explicit numerical approximation of Kähler-Einstein metric of projective manifolds, Donaldson introduced in [3] various operators with good properties to approximate classical operators. See the discussions in Section 4.2 of [3] for more details related to our discussion. In this note we verify certain statement of Donaldson about the operator $Q_{K}$ in Section 4.2 by using the full asymptotic expansion of Bergman kernel derived in $[2$, Theorem 4.18] and $[4, \S 3.4]$. Such statement is needed for the convergence of the approximation procedure.

As a warm up, we explain first the classical Bergman kernel on $\mathbb{C}^{n}[4$, Remark 1.14] which will serve as a model for our problem.

Let $F=\mathbb{C}$ be the trivial holomorphic line bundle on $\mathbb{C}^{n}$ with the canonical section 1. Let $h^{F}$ be the metric on $F$ defined by $|\mathbf{1}|_{h^{F}}(z):=e^{-\frac{\pi}{2}|Z|^{2}}$ for $z \in \mathbb{C}^{n}$ with $|Z|^{2}=\sum_{j=1}^{n}\left|z_{j}\right|^{2}$. Let $g^{T \mathbb{C}^{n}}$ be the Euclidean metric on $\mathbb{C}^{n}$. Let $P$ be the orthogonal projection from $\left(L^{2}\left(\mathbb{C}^{n}, F\right),\|\|_{L^{2}}\right)$ onto the space of $L^{2}$-holomorphic sections of $F$, and let $P\left(z, z^{\prime}\right)\left(z, z^{\prime} \in \mathbb{C}^{n}\right)$ be the smooth kernel of $P$ with respect to the Euclidean volume form $d Z$. We trivialize $F$ by using the unit section $e^{\frac{\pi}{2}|Z|^{2}} \mathbf{1}$. Then an orthonormal basis of $L^{2}$-holomorphic sections of $F$ under this trivialization is

$$
\left(\frac{(2 \pi)^{\beta}}{2^{|\beta|} \beta !}\right)^{1 / 2} z^{\beta} \exp \left(-\frac{\pi}{2}|Z|^{2}\right), \quad \beta \in \mathbb{N}^{n},
$$

and the classical Bergman kernel $P\left(z, z^{\prime}\right)$ (cf. $\left.[2,(4.114)],[4,(1.91)]\right)$, is

$$
P\left(z, z^{\prime}\right)=\exp \left(-\frac{\pi}{2} \sum_{i=1}^{n}\left(\left|z_{i}\right|^{2}+\left|z_{i}^{\prime}\right|^{2}-2 z_{i} \bar{z}_{i}^{\prime}\right)\right) \text {. }
$$

Recall that the classical heat kernel on $\mathbb{C}^{n}$ is $e^{-u \Delta}\left(z, z^{\prime}\right)=(4 \pi u)^{-n} e^{-\frac{1}{4 u}\left|Z-Z^{\prime}\right|^{2}}$. Thus from (2), we get

$$
\left|P\left(z, z^{\prime}\right)\right|^{2}=e^{-\pi\left|Z-Z^{\prime}\right|^{2}}=e^{-\frac{\Delta}{4 \pi}}\left(z, z^{\prime}\right) .
$$

In this note, we will establish an asymptotic version of (3) in the general case.

Let $(X, \omega, J)$ be a compact Kähler manifold of $\operatorname{dim}_{\mathbb{C}} X=n$, and let $\left(L, h^{L}\right)$ be a holomorphic Hermitian line bundle on $X$. Let $\nabla^{L}$ be the holomorphic Hermitian

Received by the editors February 20, 2006. 
connection on $\left(L, h^{L}\right)$ with curvature $R^{L}$. We assume that

$$
\frac{\sqrt{-1}}{2 \pi} R^{L}=\omega
$$

Let $g^{T X}(\cdot, \cdot):=\omega(\cdot, J \cdot)$ be the Riemannian metric on $T X$ induced by $\omega, J$. Let $d v_{X}$ be the Riemannian volume form of $\left(T X, g^{T X}\right)$, then $d v_{X}=\omega^{n} / n$ !. Let $d \nu$ be any volume form on $X$. Let $\eta$ be the positive function on $X$ defined by

$$
d v_{X}=\eta d \nu
$$

The $L^{2}$-scalar product \langle\rangle$_{\nu}$ on $\mathscr{C}^{\infty}\left(X, L^{p}\right)$, the space of smooth sections of $L^{p}$, is given by

$$
\left\langle\sigma_{1}, \sigma_{2}\right\rangle_{\nu}:=\int_{X}\left\langle\sigma_{1}(x), \sigma_{2}(x)\right\rangle_{h^{L^{p}}} d \nu(x) .
$$

Let $P_{\nu, p}\left(x, x^{\prime}\right)\left(x, x^{\prime} \in X\right)$ be the smooth kernel of the orthogonal projection from $\left(\mathscr{C}^{\infty}\left(X, L^{p}\right),\langle\rangle_{\nu}\right)$ onto $H^{0}\left(X, L^{p}\right)$, the space of the holomorphic sections of $L^{p}$ on $X$, with respect to $d \nu\left(x^{\prime}\right)$. Note that $P_{\nu, p}\left(x, x^{\prime}\right) \in L_{x}^{p} \otimes L_{x^{\prime}}^{p *}$. Following [3, §4], set

$$
K_{p}\left(x, x^{\prime}\right):=\left|P_{\nu, p}\left(x, x^{\prime}\right)\right|_{h_{x}^{L p} \otimes h_{x^{\prime}}^{L^{p *}}}^{2}, \quad R_{p}:=\left(\operatorname{dim} H^{0}\left(X, L^{p}\right)\right) / \operatorname{Vol}(X, \nu),
$$

here $\operatorname{Vol}(X, \nu):=\int_{X} d \nu . \operatorname{Set} \operatorname{Vol}\left(X, d v_{X}\right):=\int_{X} d v_{X}$.

Let $Q_{K_{p}}$ be the integral operator associated to $K_{p}$ which is defined for $f \in \mathscr{C}^{\infty}(X)$,

$$
Q_{K_{p}}(f)(x):=\frac{1}{R_{p}} \int_{X} K_{p}(x, y) f(y) d \nu(y) .
$$

Let $\Delta$ be the (positive) Laplace operator on $\left(X, g^{T X}\right)$ acting on the functions on $X$. We denote by ||$_{L^{2}}$ the $L^{2}$-norm on the function on $X$ with respect to $d v_{X}$.

Theorem 0.1. There exists a constant $C>0$ such that for any $f \in \mathscr{C}^{\infty}(X), p \in \mathbb{N}$,

$$
\begin{aligned}
& \left|\left(Q_{K_{p}}-\frac{\operatorname{Vol}(X, \nu)}{\operatorname{Vol}\left(X, d v_{X}\right)} \eta \exp \left(-\frac{\Delta}{4 \pi p}\right)\right) f\right|_{L^{2}} \leq \frac{C}{p}|f|_{L^{2}}, \\
& \left|\left(\frac{\Delta}{p} Q_{K_{p}}-\frac{\operatorname{Vol}(X, \nu)}{\operatorname{Vol}\left(X, d v_{X}\right)} \frac{\Delta}{p} \eta \exp \left(-\frac{\Delta}{4 \pi p}\right)\right) f\right|_{L^{2}} \leq \frac{C}{p}|f|_{L^{2}} .
\end{aligned}
$$

Moreover, (9) is uniform in that there is an integer $s$ such that if all data $h^{L}, d \nu$ run over a set which is bounded in $\mathscr{C}^{s}$-topology and that $g^{T X}, d v_{X}$ are bounded from below, then the constant $C$ is independent of $h^{L}, d \nu$.

Proof. We explain at first the full asymptotic expansion of $P_{\nu, p}\left(x, x^{\prime}\right)$ from [2, Theorem 4.18'] and $[4, \S 3.4]$. For more details on our approach we also refer the readers to the recent book [5].

Let $E=\mathbb{C}$ be the trivial holomorphic line bundle on $X$. Let $h^{E}$ the metric on $E$ defined by $|\mathbf{e}|_{h^{E}}^{2}=1$, here $\mathbf{e}$ is the canonical unity element of $E$. We identify canonically $L^{p}$ to $L^{p} \otimes E$ by section $\mathbf{e}$.

As in $[4, \S 3.4]$, let $h_{\omega}^{E}$ be the metric on $E$ defined by $|\mathbf{e}|_{h_{\omega}^{E}}^{2}=\eta^{-1}$. Let \langle\rangle$_{\omega}$ be the Hermitian product on $\mathscr{C}^{\infty}\left(X, L^{p} \otimes E\right)=\mathscr{C}^{\infty}\left(X, L^{p}\right)$ induced by $h^{L}, h_{\omega}^{E}, d v_{X}$ as in (6). Then by (5),

$$
\left(\mathscr{C}^{\infty}\left(X, L^{p} \otimes E\right),\langle\quad\rangle_{\omega}\right)=\left(\mathscr{C}^{\infty}\left(X, L^{p}\right),\langle\quad\rangle_{\nu}\right) .
$$


Observe that $H^{0}\left(X, L^{p} \otimes E\right)$ does not depend on $g^{T X}, h^{L}$ or $h^{E}$. If $P_{\omega, p}\left(x, x^{\prime}\right)$, $\left(x, x^{\prime} \in X\right)$ denotes the smooth kernel of the orthogonal projection $P_{\omega, p}$ from $\left(\mathscr{C}^{\infty}\left(X, L^{p} \otimes E\right),\langle\cdot, \cdot\rangle_{\omega}\right)$ onto $H^{0}\left(X, L^{p} \otimes E\right)=H^{0}\left(X, L^{p}\right)$ with respect to $d v_{X}(x)$, from $(5)$, as in $[4,(3.38)]$, we have

$$
P_{\nu, p}\left(x, x^{\prime}\right)=\eta\left(x^{\prime}\right) P_{\omega, p}\left(x, x^{\prime}\right) .
$$

For $f \in \mathscr{C}^{\infty}(X)$, set

$$
\begin{aligned}
& K_{\omega, p}\left(x, x^{\prime}\right)=\left|P_{\omega, p}\left(x, x^{\prime}\right)\right|_{\left(h^{L^{p}} \otimes h_{\omega}^{E}\right)_{x} \otimes\left(h^{L^{p *}} \otimes h_{\omega}^{E^{*}}\right)_{x^{\prime}}}^{2}, \\
& \left(K_{\omega, p} f\right)(x)=\int_{X} K_{\omega, p}(x, y) f(y) d v_{X}(y) .
\end{aligned}
$$

By the definition of the metric $h^{E}, h_{\omega}^{E}$, if we denote by $\mathbf{e}^{*}$ the dual of the section e of $E$, we know

$$
1=\left|\mathbf{e} \otimes \mathbf{e}^{*}\right|_{h^{E} \otimes h^{E^{*}}}^{2}\left(x, x^{\prime}\right)=\left|\mathbf{e} \otimes \mathbf{e}^{*}\right|_{h_{\omega}^{E} \otimes h_{\omega}^{E^{*}}}^{2}\left(x, x^{\prime}\right) \eta(x) \eta^{-1}\left(x^{\prime}\right) .
$$

Recall that we identified $\left(L^{p}, h^{L^{p}}\right)$ to $\left(L^{p} \otimes E, h^{L^{p}} \otimes h^{E}\right)$ by section e. Thus from (7), (11) and (13), we get

$$
K_{p}\left(x, x^{\prime}\right)=\left|P_{\nu, p}\left(x, x^{\prime}\right)\right|_{\left(h^{L^{p}} \otimes h^{E}\right)_{x} \otimes\left(h^{L^{p *}} \otimes h^{E^{*}}\right)_{x^{\prime}}}^{2}=\eta(x) \eta\left(x^{\prime}\right) K_{\omega, p}\left(x, x^{\prime}\right),
$$

and from (5), (8) and (14),

$$
Q_{K_{p}}(f)(x)=\frac{1}{R_{p}} \int_{X} K_{\omega, p}(x, y) \eta(x) f(y) d v_{X}(y) .
$$

Now for the kernel $P_{\omega, p}\left(x, x^{\prime}\right)$, we can apply the full asymptotic expansion [2, Theorem 4.18']. In fact let $\bar{\partial}^{L^{p} \otimes E, *_{\omega}}$ be the formal adjoint of the Dolbeault operator $\bar{\partial}^{L^{p} \otimes E}$ on the Dolbeault complex $\Omega^{0, \bullet}\left(X, L^{p} \otimes E\right)$ with the scalar product induced by $g^{T X}, h^{L}, h_{\omega}^{E}, d v_{X}$ as in (6), and set

$$
D_{p}=\sqrt{2}\left(\bar{\partial}^{L^{p} \otimes E}+\bar{\partial}^{L^{p} \otimes E, * \omega}\right) .
$$

Then $H^{0}\left(X, L^{p} \otimes E\right)=\operatorname{Ker}\left(D_{p}\right)$ for $p$ large enough, and $D_{p}$ is a Dirac operator, as $g^{T X}(\cdot, \cdot)=\omega(\cdot, J \cdot)$ is a Kähler metric on $T X$.

Let $\nabla^{E}$ be the holomorphic Hermitian connection on $\left(E, h_{\omega}^{E}\right)$. Let $\nabla^{T X}$ be the Levi-Civita connection on $\left(T X, g^{T X}\right)$. Let $R^{E}, R^{T X}$ be the corresponding curvatures.

Let $d(x, y)$ be the Riemannian distance from $x$ to $y$ on $\left(X, g^{T X}\right)$. Let $a^{X}$ be the injectivity radius of $\left(X, g^{T X}\right)$. We fix $\left.\varepsilon \in\right] 0, a^{X} / 4\left[\right.$. We denote by $B^{X}(x, \varepsilon)$ and $B^{T_{x} X}(0, \varepsilon)$ the open balls in $X$ and $T_{x} X$ with center $x$ and radius $\varepsilon$. We identify $B^{T_{x} X}(0, \varepsilon)$ with $B^{X}(x, \varepsilon)$ by using the exponential map of $\left(X, g^{T X}\right)$.

We fix $x_{0} \in X$. For $Z \in B^{T_{x_{0}} X}(0, \varepsilon)$ we identify $\left(L_{Z}, h_{Z}^{L}\right),\left(E_{Z}, h_{Z}^{E}\right)$ and $\left(L^{p} \otimes E\right)_{Z}$ to $\left(L_{x_{0}}, h_{x_{0}}^{L}\right),\left(E_{x_{0}}, h_{x_{0}}^{E}\right)$ and $\left(L^{p} \otimes E\right)_{x_{0}}$ by parallel transport with respect to the connections $\nabla^{L}, \nabla^{E}$ and $\nabla^{L^{p} \otimes E}$ along the curve $\gamma_{Z}:[0,1] \ni u \rightarrow \exp _{x_{0}}^{X}(u Z)$. Then under our identification, $P_{\omega, p}\left(Z, Z^{\prime}\right)$ is a function on $Z, Z^{\prime} \in T_{x_{0}} X,|Z|,\left|Z^{\prime}\right| \leq \varepsilon$, we denote it by $P_{\omega, p, x_{0}}\left(Z, Z^{\prime}\right)$.

Let $\pi: T X \times_{X} T X \rightarrow X$ be the natural projection from the fiberwise product of $T X$ on $X$. Then we can view $P_{\omega, p, x_{0}}\left(Z, Z^{\prime}\right)$ as a smooth function on $T X \times_{X} T X$ with complex values (which is defined for $|Z|,\left|Z^{\prime}\right| \leq \varepsilon$ ) by identifying a section $S \in$ 
$\mathscr{C}^{\infty}\left(T X \times_{X} T X, \pi^{*} \operatorname{End}(E)\right)$ with the family $\left(S_{x}\right)_{x \in X}$, where $S_{x}=\left.S\right|_{\pi^{-1}(x)}$, since $\operatorname{End}(E)=\mathbb{C}$.

We choose $\left\{w_{i}\right\}_{i=1}^{n}$ an orthonormal basis of $T_{x_{0}}^{(1,0)} X$, then $e_{2 j-1}=\frac{1}{\sqrt{2}}\left(w_{j}+\bar{w}_{j}\right)$ and $e_{2 j}=\frac{\sqrt{-1}}{\sqrt{2}}\left(w_{j}-\bar{w}_{j}\right), j=1, \ldots, n$ forms an orthonormal basis of $T_{x_{0}} X$. We use the coordinates on $T_{x_{0}} X \simeq \mathbb{R}^{2 n}$ where the identification is given by

$$
\left(Z_{1}, \cdots, Z_{2 n}\right) \in \mathbb{R}^{2 n} \longrightarrow \sum_{i=1}^{2 n} Z_{i} e_{i} \in T_{x_{0}} X .
$$

In what follows we also introduce the complex coordinates $z=\left(z_{1}, \cdots, z_{n}\right)$ on $\mathbb{C}^{n} \simeq$ $\mathbb{R}^{2 n}$.

By [2, Proposition 4.1], for any $l, m \in \mathbb{N}, \varepsilon>0$, there exists $C_{l, m, \varepsilon}>0$ such that for $p \geq 1, x, x^{\prime} \in X$,

$$
\left|P_{\omega, p}\left(x, x^{\prime}\right)\right|_{\mathscr{C}^{m}(X \times X)} \leq C_{l, m, \varepsilon} p^{-l} \quad \text { if } d\left(x, x^{\prime}\right) \geq \varepsilon .
$$

Here the $\mathscr{C}^{m}$-norm is induced by $\nabla^{L}, \nabla^{E}, \nabla^{T X}$ and $h^{L}, h^{E}, g^{T X}$.

By $\left[2\right.$, Theorem $\left.4.18^{\prime}\right]$, there exist $J_{r}\left(Z, Z^{\prime}\right)$ polynomials in $Z, Z^{\prime}$, such that for any $k, m, m^{\prime} \in \mathbb{N}$, there exist $N \in \mathbb{N}, C>0, C_{0}>0$ such that for $\alpha, \alpha^{\prime} \in \mathbb{N}^{n},|\alpha|+\left|\alpha^{\prime}\right| \leq m$, $Z, Z^{\prime} \in T_{x_{0}} X,|Z|,\left|Z^{\prime}\right| \leq \varepsilon, x_{0} \in X, p>1$,

$$
\begin{aligned}
& \left|\frac{\partial^{|\alpha|+\left|\alpha^{\prime}\right|}}{\partial Z^{\alpha} \partial Z^{\prime \alpha^{\prime}}}\left(\frac{1}{p^{n}} P_{\omega, p, x_{0}}\left(Z, Z^{\prime}\right)-\sum_{r=0}^{k}\left(J_{r} P\right)\left(\sqrt{p} Z, \sqrt{p} Z^{\prime}\right) p^{-r / 2}\right)\right|_{\mathscr{C} m^{\prime}(X)} \\
& \quad \leq C p^{-(k+1-m) / 2}\left(1+|\sqrt{p} Z|+\left|\sqrt{p} Z^{\prime}\right|\right)^{N} \exp \left(-C_{0} \sqrt{p}\left|Z-Z^{\prime}\right|\right)+\mathscr{O}\left(p^{-\infty}\right) .
\end{aligned}
$$

Here $\mathscr{C}^{m^{\prime}}(X)$ is the $\mathscr{C}^{m^{\prime}}$ norm for the parameter $x_{0} \in X$. The term $\mathscr{O}\left(p^{-\infty}\right)$ means that for any $l, l_{1} \in \mathbb{N}$, there exists $C_{l, l_{1}}>0$ such that its $\mathscr{C}^{l_{1}}$-norm is dominated by $C_{l, l_{1}} p^{-l}$.

Now we claim that in (19),

$$
J_{0}=1, \quad J_{1}\left(Z, Z^{\prime}\right)=0 .
$$

In fact, let $d v_{T_{x_{0}} X}$ be the Riemannian volume form on $\left(T_{x_{0}} X, g^{T_{x_{0}} X}\right)$, and $\kappa_{x_{0}}$ be the function defined by

$$
d v_{X}(Z)=\kappa_{x_{0}}(Z) d v_{T_{x_{0}} X}(Z)
$$

Then (also cf. $[4,(1.31)])$

$$
\kappa_{x_{0}}(Z)=1+\frac{1}{6}\left\langle R_{x_{0}}^{T X}\left(Z, e_{i}\right) Z, e_{i}\right\rangle_{x_{0}}+\mathscr{O}\left(|Z|^{3}\right) .
$$

As we only work on $\mathscr{C}^{\infty}\left(X, L^{p} \otimes E\right)$, by [2, (4.115)], we get the first equation in (20).

Recall that in the normal coordinate, after the rescaling $Z \rightarrow Z / t$ with $t=\frac{1}{\sqrt{p}}$, we get an operator $\mathscr{L}_{t}$ from the restriction of $D_{p}^{2}$ on $\mathscr{C}^{\infty}\left(X, L^{p} \otimes E\right)$ which has the following formal expansion (cf. [2, (4.104)], [4, Theorem 1.4]),

$$
\mathscr{L}_{t}=\mathscr{L}+\sum_{r=1}^{\infty} \mathcal{Q}_{r} t^{r}
$$


Now, from $[2$, Theorem 5.1] (or $[4,(1.87),(1.98)]$ ),

$$
\mathscr{L}=\sum_{j=1}^{n}\left(-2 \frac{\partial}{\partial z_{i}}+\pi \bar{z}_{i}\right)\left(2 \frac{\partial}{\partial \bar{z}_{i}}+\pi z_{i}\right), \quad \mathcal{Q}_{1}=0 .
$$

(In fact, $P\left(Z, Z^{\prime}\right)$ is the smooth kernel of the orthogonal projection from $L^{2}\left(\mathbb{C}^{n}\right)$ onto $\operatorname{Ker}(\mathscr{L})$ ). Thus from $[2,(4.107)]$ (cf. $[4,(1.111)]),(22)$ and $(24)$ we get the second equation of (20).

Note that $\left|P_{\omega, p, x_{0}}\left(Z, Z^{\prime}\right)\right|^{2}=P_{\omega, p, x_{0}}\left(Z, Z^{\prime}\right) \overline{P_{\omega, p, x_{0}}\left(Z, Z^{\prime}\right)}$, thus from (12), (19) and (20), there exist $J_{r}^{\prime}\left(Z, Z^{\prime}\right)$ polynomials in $Z, Z^{\prime}$ such that

$$
\begin{aligned}
& \mid \frac{1}{p^{2 n+1}} \Delta_{Z}\left.\left(K_{\omega, p, x_{0}}\left(Z, Z^{\prime}\right)-\left(1+\sum_{r=2}^{k} p^{-r / 2} J_{r}^{\prime}\left(\sqrt{p} Z, \sqrt{p} Z^{\prime}\right)\right) e^{-\pi p\left|Z-Z^{\prime}\right|^{2}}\right)\right|_{\mathscr{C}^{0}(X)} \\
& \leq C p^{-(k+1) / 2}\left(1+|\sqrt{p} Z|+\left|\sqrt{p} Z^{\prime}\right|\right)^{N} \exp \left(-C_{0} \sqrt{p}\left|Z-Z^{\prime}\right|\right)+\mathscr{O}\left(p^{-\infty}\right) .
\end{aligned}
$$

For a function $f \in \mathscr{C}^{\infty}(X)$, we denote it as $f_{x_{0}}(Z)$ a family (with parameter $x_{0}$ ) of function of $Z$ in the normal coordinate near $x_{0}$. Now, for any polynomial $A_{x_{0}}\left(Z^{\prime}\right)$, we define the operator

$$
\left(\mathcal{A}_{p} f\right)\left(x_{0}\right)=p^{n} \int_{\left|Z^{\prime}\right| \leq \varepsilon} A_{x_{0}}\left(\sqrt{p} Z^{\prime}\right) e^{-\pi p\left|Z^{\prime}\right|^{2}} f_{x_{0}}\left(Z^{\prime}\right) d v_{X}\left(Z^{\prime}\right) .
$$

Then we observe that there exists $C_{1}>0$ such that for any $p \in \mathbb{N}, f \in \mathscr{C}^{\infty}(X)$, we have

$$
\left|\mathcal{A}_{p} f\right|_{L^{2}} \leq C_{1}|f|_{L^{2}} .
$$

In fact, there exist $C^{\prime}, C_{1}>0$ independent on $p$ such that

$$
\begin{aligned}
& \left|\mathcal{A}_{p} f\right|_{L^{2}}^{2} \leq \int_{X} d v_{X}\left(x_{0}\right)\left\{p^{n}\left(\int_{\left|Z^{\prime}\right| \leq \varepsilon}\left|A_{x_{0}}\left(\sqrt{p} Z^{\prime}\right)\right| e^{-\pi p\left|Z^{\prime}\right|^{2}} d v_{X}\left(Z^{\prime}\right)\right)\right. \\
& \left.\times p^{n}\left(\int_{\left|Z^{\prime}\right| \leq \varepsilon}\left|A_{x_{0}}\left(\sqrt{p} Z^{\prime}\right)\right| e^{-\pi p\left|Z^{\prime}\right|^{2}}\left|f_{x_{0}}\left(Z^{\prime}\right)\right|^{2} d v_{X}\left(Z^{\prime}\right)\right)\right\} \\
& \leq C^{\prime} \int_{X} d v_{X}\left(x_{0}\right) p^{n} \int_{\left|Z^{\prime}\right| \leq \varepsilon}\left|A_{x_{0}}\left(\sqrt{p} Z^{\prime}\right)\right| e^{-\pi p\left|Z^{\prime}\right|^{2}}\left|f_{x_{0}}\left(Z^{\prime}\right)\right|^{2} d v_{X}\left(Z^{\prime}\right) \\
& \leq C_{1}|f|_{L^{2}}^{2} .
\end{aligned}
$$

Observe that in the normal coordinate, at $Z=0, \Delta_{Z}=-\sum_{j=1}^{2 n} \frac{\partial^{2}}{\partial Z_{j}^{2}}$. Thus

$$
\left.\left(\Delta_{Z} e^{-\pi p\left|Z-Z^{\prime}\right|^{2}}\right)\right|_{Z=0}=4 \pi p\left(n-\pi p\left|Z^{\prime}\right|^{2}\right) e^{-\pi p\left|Z^{\prime}\right|^{2}} .
$$

Thus from (3), (18), (19), (20), (25) and (27), we get

$$
\begin{aligned}
& \left|p^{-n} K_{\omega, p} f-p^{n} \int_{\left|Z^{\prime}\right| \leq \varepsilon} e^{-\pi p\left|Z^{\prime}\right|^{2}} f_{x_{0}}\left(Z^{\prime}\right) d v_{X}\left(Z^{\prime}\right)\right|_{L^{2}} \leq \frac{C}{p}|f|_{L^{2}}, \\
& \left|p^{-n-1} \Delta K_{\omega, p} f-4 \pi p^{n} \int_{\left|Z^{\prime}\right| \leq \varepsilon}\left(n-\pi p\left|Z^{\prime}\right|^{2}\right) e^{-\pi p\left|Z^{\prime}\right|^{2}} f_{x_{0}}\left(Z^{\prime}\right) d v_{X}\left(Z^{\prime}\right)\right|_{L^{2}} \leq \frac{C}{p}|f|_{L^{2}} .
\end{aligned}
$$


Recall that $\eta \in \mathscr{C}^{\infty}(X)$ was defined in (5). Set

$$
\begin{aligned}
& K_{\eta, \omega, p}(x, y)=\left\langle d \eta(x), d_{x} K_{\omega, p}(x, y)\right\rangle_{g^{T^{*} X}}, \\
& \left(K_{\eta, \omega, p} f\right)(x)=\int_{X} K_{\eta, \omega, p}(x, y) f(y) d v_{X}(y) .
\end{aligned}
$$

Then from (19), (20) and (27), we get

$\left|p^{-n-1} K_{\eta, \omega, p} f-2 \pi p^{n} \int_{\left|Z^{\prime}\right| \leq \varepsilon} \sum_{i=1}^{2 n}\left(\frac{\partial}{\partial Z_{i}} \eta\right)\left(x_{0}, 0\right) Z_{i}^{\prime} e^{-\pi p\left|Z^{\prime}\right|^{2}} f_{x_{0}}\left(Z^{\prime}\right) d v_{X}\left(Z^{\prime}\right)\right|_{L^{2}} \leq \frac{C}{p}|f|_{L^{2}}$,

here $C$ is taken large enough so that both (30) and (32) hold and is independent on $p$.

Let $e^{-u \Delta}\left(x, x^{\prime}\right)$ be the smooth kernel of the heat operator $e^{-u \Delta}$ with respect to $d v_{X}\left(x^{\prime}\right)$. By the heat kernel expansion in [1, Theorems 2.23, 2.26], there exist $\Phi_{i}(x, y)$ smooth functions on $X \times X$ such that when $u \rightarrow 0$, we have the following asymptotic expansion

$$
\left|\frac{\partial^{l}}{\partial u^{l}}\left(e^{-u \Delta}(x, y)-(4 \pi u)^{-n} \sum_{i=0}^{k} u^{i} \Phi_{i}(x, y) e^{-\frac{1}{4 u} d(x, y)^{2}}\right)\right|_{\mathscr{C}^{m}(X \times X)}=\mathscr{O}\left(u^{k-n-l-\frac{m}{2}+1}\right),
$$

and

$$
\Phi_{0}(x, y)=1 .
$$

If we still use the normal coordinate, then by (33), there exist $\phi_{i, x_{0}}\left(Z^{\prime}\right):=\Phi_{i}\left(0, Z^{\prime}\right)$ such that uniformly for $x_{0} \in X, Z^{\prime} \in T_{x_{0}} X,\left|Z^{\prime}\right| \leq \varepsilon$, we have the following asymptotic expansion when $u \rightarrow 0$,

$$
\left|\frac{\partial^{l}}{\partial u^{l}}\left(e^{-u \Delta}\left(0, Z^{\prime}\right)-(4 \pi u)^{-n}\left(1+\sum_{i=1}^{k} u^{i} \phi_{i, x_{0}}\left(Z^{\prime}\right)\right) e^{-\frac{1}{4 u}\left|Z^{\prime}\right|^{2}}\right)\right|_{\mathscr{C}^{0}(X)}=\mathscr{O}\left(u^{k-n-l+1}\right),
$$

and

$$
\begin{aligned}
& \mid\left\langle d \eta\left(x_{0}\right), d_{x_{0}} e^{-u \Delta}\right\rangle_{g^{T^{*} X}}\left(0, Z^{\prime}\right) \\
& \left.\quad-(4 \pi u)^{-n} \sum_{i=1}^{2 n}\left(\frac{\partial}{\partial Z_{i}} \eta\right)\left(x_{0}, 0\right) \frac{Z_{i}^{\prime}}{2 u}\left(1+\sum_{i=1}^{k} u^{i} \phi_{i, x_{0}}\left(Z^{\prime}\right)\right)\right) e^{-\frac{1}{4 u}\left|Z^{\prime}\right|^{2}} \\
& \quad-\left.(4 \pi u)^{-n} \sum_{i=1}^{k} u^{i}\left\langle d \eta\left(x_{0}\right),\left(d_{x_{0}} \Phi_{i}\right)\left(0, Z^{\prime}\right)\right\rangle e^{-\frac{1}{4 u}\left|Z^{\prime}\right|^{2}}\right|_{\mathscr{C}^{0}(X)}=\mathscr{O}\left(u^{k-n+\frac{1}{2}}\right) .
\end{aligned}
$$

Observe that

$$
\frac{1}{p} \Delta \exp \left(-\frac{\Delta}{4 \pi p}\right)=-\left.\frac{1}{p}\left(\frac{\partial}{\partial u} e^{-u \Delta}\right)\right|_{u=\frac{1}{4 \pi p}}
$$


Now from (27), (30)-(37) with $k=n+1$, we get

$$
\begin{aligned}
& \left|\left(p^{-n} K_{\omega, p}-\exp \left(-\frac{\Delta}{4 \pi p}\right)\right) f\right|_{L^{2}} \leq \frac{C}{p}|f|_{L^{2}}, \\
& \left|\frac{1}{p}\left(p^{-n} \Delta K_{\omega, p}-\Delta \exp \left(-\frac{\Delta}{4 \pi p}\right)\right) f\right|_{L^{2}} \leq \frac{C}{p}|f|_{L^{2}} .
\end{aligned}
$$

and

$$
\left|\frac{1}{p}\left(p^{-n} K_{\eta, \omega, p}-\left\langle d \eta, d \exp \left(-\frac{\Delta}{4 \pi p}\right)\right\rangle\right) f\right|_{L^{2}} \leq \frac{C}{p}|f|_{L^{2}} .
$$

Note that

$$
\begin{aligned}
\left(\Delta\left(\eta K_{\omega, p}\right)\right)(x, y)=(\Delta \eta)(x) K_{\omega, p}(x, y)+\eta(x) \Delta_{x} K_{\omega, p}(x, y) & \\
& -2\left\langle d \eta(x), d_{x} K_{\omega, p}(x, y)\right\rangle_{g^{T^{*} X}}
\end{aligned}
$$

and $R_{p}=\frac{\operatorname{Vol}\left(X, d v_{X}\right)}{\operatorname{Vol}(X, \nu)} p^{n}+\mathscr{O}\left(p^{n-1}\right)$. From (15), (38)-(40), we get (9).

To get the last part of Theorem 0.1 , as we noticed in $[2, \S 4.5]$, the constants in (19) will be uniformly bounded under our condition, thus we can take $C$ in (9), (38)and (39) independent of $h^{L}, d \nu$.

\section{Acknowledgments}

We thank Professor Simon Donaldson for useful communications. We are indebted to a referee for his very careful reading of the manuscript, and also for useful comments.

\section{References}

[1] N. Berline, E. Getzler, and M. Vergne, Heat kernels and Dirac operators, Springer-Verlag, Berlin, 1992.

[2] X. Dai, K. Liu, and X. Ma, On the asymptotic expansion of Bergman kernel, J. Differential Geom. 72 (2006), no. 1, 1-41. announced in C. R. Math. Acad. Sci. Paris 339 (2004), no. 3, 193-198.

[3] S. K. Donaldson, Some numerical results in complex differential geometry, math.DG/0512625.

[4] X. Ma and G. Marinescu, Generalized Bergman kernels on symplectic manifolds, C. R. Math. Acad. Sci. Paris 339 (2004), no. 7, 493-498. The full version: math.DG/0411559.

[5] __ Holomorphic Morse Inequalities and Bergman Kernels, Progress in Mathematics 254, Birkhäuser Boston, Inc., Boston, MA 2007.

Center of Mathematical Science, Zhejiang University and Department of Mathematics, UCLA, CA 90095-1555, USA

E-mail address: liu@math.ucla.edu

Centre de Mathématiques Laurent Schwartz, UMR 7640 du CNRS, Ecole Polytechnique, 91128 Palaiseau Cedex, France

E-mail address: ma@math.polytechnique.fr 